\title{
Analysis of the Effects of Finite Precision in Neural Network-Based Sound Classifiers for Digital Hearing Aids
}

\author{
Roberto Gil-Pita (EURASIP Member), Enrique Alexandre, Lucas Cuadra \\ (EURASIP Member), Raúl Vicen, and Manuel Rosa-Zurera (EURASIP Member)
}

\author{
Departamento de Teoría de la Señal y Comunicaciones, Escuela Politécnica Superior, Universidad de Alcalá, \\ 28805 Alcala de Henares, Spain \\ Correspondence should be addressed to Roberto Gil-Pita, roberto.gil@uah.es
}

Received 1 December 2008; Revised 4 May 2009; Accepted 9 September 2009

Recommended by Hugo Fastl

\begin{abstract}
The feasible implementation of signal processing techniques on hearing aids is constrained by the finite precision required to represent numbers and by the limited number of instructions per second to implement the algorithms on the digital signal processor the hearing aid is based on. This adversely limits the design of a neural network-based classifier embedded in the hearing aid. Aiming at helping the processor achieve accurate enough results, and in the effort of reducing the number of instructions per second, this paper focuses on exploring (1) the most appropriate quantization scheme and (2) the most adequate approximations for the activation function. The experimental work proves that the quantized, approximated, neural network-based classifier achieves the same efficiency as that reached by "exact" networks (without these approximations), but, this is the crucial point, with the added advantage of extremely reducing the computational cost on the digital signal processor.
\end{abstract}

Copyright ( $) 2009$ Roberto Gil-Pita et al. This is an open access article distributed under the Creative Commons Attribution License, which permits unrestricted use, distribution, and reproduction in any medium, provided the original work is properly cited.

\section{Introduction}

This paper focuses on exploring to what extent the use of a quantized, approximated neural network-(NN-) based classifier embedded in a digital hearing aid could appreciably affect the performance of this device. This phrase probably makes the reader not directly involved in hearing aid design wonder.

(1) Why do the authors propose a hearing aid capable of classifying sounds?

(2) Why do they propose a neural network for classifying (if there are more simple solutions)?

(3) Why do they study the effects associated with quantizing and approximating it? Are these effects so important?

The first question is related to the fact that hearing aid users usually face a variety of sound environments. A hearing aid capable of automatically classifying the acoustic environment that surrounds his/her user, and selecting the amplification "program" that is best adapted to such environment ("self-adaptation") would improve the user's comfort [1]. The "manual" approach, in which the user has to identify the acoustic surroundings, and to choose the adequate program, is very uncomfortable and frequently exceeds the abilities of many hearing aid users [2]. This illustrates the necessity for hearing aids to automatically classify the acoustic environment the user is in [3].

Furthermore, sound classification is also used in modern hearing aids as a support for the noise reduction and source separation stages, like, for example, in voice activity detection (VAD) [4-6]. In this case, the objective is to extract information from the sound in order to improve the performance of these systems. This second kind of classifiers differs from the first one in how often the classification is carried out. In the first case, a time scale of seconds should be enough, since it typically takes approximately $5-10$ seconds for the hearing aid user to move from one listening environment to another [7], whereas in 
the second case the information is required in shorter time slots.

The second question, related to the use of neural networks as the choice classifier, is based on the fact that neural networks exhibit very good performance when compared to other classifiers $[3,8]$, but at the expense of consuming a significantly high percentage of the available computational resources. Although difficult, the implementation of a neural network-based classifier on a hearing aid has been proven to be feasible and convenient to improve classification results [9].

Finally, regarding the latter question, the very core of our paper is motivated by the fact that the way numbers are represented is of crucial importance. The number of bits used to represent the integer and the fractional part of a number have a strong influence on the final performance of the algorithms implemented on the hearing aid, and an improper selection of these values can lead to saturations or lack of precision in the operations of the DSP. This is just one of the topics, along with the limited precision, this paper focuses on.

The problem of implementing a neural-based sound classifier in a hearing aid is that DSP-based hearing aids have constraints in terms of computational capability and memory. The hearing aid has to work at low clock rates in order to minimize the power consumption and thus maximize the battery life. Additionally, the restrictions become stronger because a considerable part of the DSP computational capabilities is already being used for running the algorithms aiming to compensate the hearing losses. Therefore, the design of any automatic sound classifier is strongly constrained to the use of the remaining resources of the DSP. This restriction in number of operations per second enforces us to put special emphasis on signal processing techniques and algorithms tailored for properly classifying while using a reduced number of operations.

Related to the aforementioned problem arises the one related to the search for the most appropriate way to implement an NN on a DSP. Most of the NNs we will be exploring consist of two layers of neurons interconnected by links with adjustable weights [10]. The way we represent such weights and the activation function of the neurons [10] may lead the classifier to fail.

Therefore, the purpose of this paper is to clearly quantify the effects of the finite-precision limitations on the performance of an automatic sound classification system for hearing aids, with special emphasis on the two aforementioned phenomena: the effects of finite word length for the weights of the NN used for the classification, and the effects of the simplification of the activation functions of the NN.

With these ideas in mind, the paper has been structured as follows. Section 2 will introduce the implemented classification system, describing the input features (Section 2.1) and the neural network (Section 2.2). Section 3 will define the considered problems: the quantization of the weights of the neural network, and use of approximations for the activation functions. Finally, Section 4 will describe the database and the protocol used for the experiments and will show the results obtained, which will be discussed in Section 5 .

\section{The System}

It basically consists of a feature extraction block and the aforementioned classifier based on a neural network.

2.1. Feature Extraction. There is a number of interesting features that could potentially exhibit different behavior for speech, music, and noise and thus may help the system classify the sound signal. In order to carry out the experiments of this paper we have selected a subset of them that provide a high discriminating capability for the problem of speech/nonspeech classification along with a considerably low associated computational cost [11]. This will assist us in testing the methods proposed in this paper. Note that the priority of the paper is not to propose these features as the best ones for all the problems considered in the paper, but to establish a set of strategies and techniques for efficiently implementing a neural network classifier in a hearing aid. We have briefly described the features below for making the paper stand by itself. The features used to characterize any sound frame are as follows.

Spectral Centroid. The spectral centroid of the $i$ th frame can be associated with the measure of brightness of the sound, and is obtained by evaluating the center of gravity of the spectrum. The centroid can be calculated by making use of the formula $[12,13]$ :

$$
\text { Centroid }_{i}=\frac{\sum_{k=1}^{K}\left|\chi_{i}(k)\right| \cdot k}{\sum_{k=1}^{K}\left|\chi_{i}(k)\right|},
$$

where $\chi_{i}(k)$ represents the $k$ th frequency bin of the spectrum at frame $i$, and $K$ is the number of samples.

Voicewhite. This parameter, proposed in [14], is a measure of the energy inside the typical speech band $(300-4000 \mathrm{~Hz}$ ) in respect to the whole energy of the signal:

$$
V 2 W_{i}=\frac{\sum_{k=M_{1}}^{M_{2}}\left|\chi_{i}(k)\right|^{2}}{\sum_{k=1}^{K}\left|\chi_{i}(k)\right|^{2}}
$$

where $M_{1}$ and $M_{2}$ are the first and the last index of the bands that are encompassed in the considered speech band.

Spectral Flux. It is associated with the amount of spectral changes over time and is defined as follows [13]:

$$
\operatorname{Flux}_{i}=\sum_{k=1}^{K}\left(\left|\chi_{i}(k)\right|-\left|\chi_{i-1}(k)\right|\right)^{2}
$$

Short Time Energy (STE). It is defined as the mean energy of the signal within each analysis frame ( $K$ samples):

$$
\mathrm{STE}_{i}=\frac{1}{K} \sum_{k=1}^{K}\left|\chi_{i}(k)\right|^{2}
$$


Finally, the features are calculated by estimating the mean value and the standard deviation of these measurements for $M$ different time frames.

$$
\mathbf{x}=\left(\begin{array}{c}
\hat{E}\left\{\text { Centroid }_{i}\right\} \\
\hat{E}\left\{V 2 W_{i}\right\} \\
\hat{E}\left\{\text { Flux }_{i}\right\} \\
\hat{E}\left\{\mathrm{STE}_{i}\right\} \\
\left(\hat{E}\left\{\text { Centroid }_{i}^{2}\right\}-\hat{E}\left\{\text { Centroid }_{i}\right\}^{2}\right)^{1 / 2} \\
\left(\hat{E}\left\{V 2 W_{i}^{2}\right\}-\hat{E}\left\{V 2 W_{i}\right\}^{2}\right)^{1 / 2} \\
\left(\hat{E}\left\{\operatorname{Flux}_{i}^{2}\right\}-\hat{E}\left\{\operatorname{Flux}_{i}\right\}^{2}\right)^{1 / 2} \\
\left(\hat{E}\left\{\operatorname{STE}_{i}^{2}\right\}-\hat{E}\left\{\operatorname{STE}_{i}\right\}^{2}\right)^{1 / 2}
\end{array}\right),
$$

where, for the sake of simplicity, we label $\widehat{E}_{i}(\cdot) \equiv$ $(1 / M) \sum_{i=1}^{M}(\cdot)$.

It is interesting to note that some of the features depend on the square amplitude of the input signal. As will be shown, the sound database includes sounds at different levels, in order to make the classification system more robust against these variations.

\subsection{Classification Algorithm}

2.2.1. Structure of a Neural Network. Figure 1 shows a simple Multilayer Perceptron (MLP) with $L=8$ inputs, $N=2$ hidden neurons and $C=3$ outputs, interconnected by links with adjustable weights. Each neuron applies a linear combination of its inputs to a nonlinear function called activation function. In our case, the model of each neuron includes a nonlinear activation function (the hyperbolic tangent function), which can be calculated using the following expression:

$$
f(x)=\tanh (x)=\frac{e^{x}-e^{-x}}{e^{x}+e^{-x}} .
$$

From the expression above it is straightforward to see that implementing this function on the hearing aid DSP is not an easy task, since an exponential and a division need to be computed. This motivates the need for exploring simplifications of this activation function that could provide similar results in terms of probability of error.

The number of neurons in the input and the output layers seems to be clear: the input neurons $(L)$ represent the components of the feature vector and thus, and its dimension will depend on the number of features used in each experiment. On the other hand, the number of the neurons in the output layer $(C)$ is determined by the number of audio classes to classify, speech, music or noise.

The network also contains one layer of $N$ hidden neurons that is not part of the input or output of the network. These $N$ hidden neurons enable the network to learn complex tasks by extracting progressively more meaningful features from the input vectors. But, what is the optimum numbers of hidden neurons $N$ ? The answer to this question is related to the adjustment of the complexity of the network [10]. If too many free weights are used, the capability to generalize will be poor; on the contrary if too few parameters are considered, the training data cannot be learned satisfactorily.

One important fact that must be considered in the implementation of an MLP is that a scale factor in one of the inputs $\left(x_{n}^{\prime}=x_{n} k\right)$ can be compensated with a change in the corresponding weights of the hidden layer $\left(v_{n m}^{\prime}=v_{n m} / k\right.$, for $m=1, \ldots, L)$, so that the outputs of the linear combinations $\left(a_{m}\right)$ are not affected $\left(v_{n m}^{\prime} x_{n}^{\prime}=v_{n m} x_{n}\right)$. This fact is important, since it allows scaling each feature so that it uses the entire dynamic range of the numerical representation, minimizing the effects of the finite precision over the features without affecting the final performance of the neural network.

Another important property of the MLP is related to the output of the network. Considering that the activation function is a monotonically increasing function, if $z_{i}>z_{j}$, then $b_{i}>b_{j}$. Therefore, since the final decision is taken by comparing the outputs of the neural network and looking for the greatest value, once the network is trained there is no need of determining the complete output of the network $\left(z_{i}\right)$, being enough to determine the linear combinations of the output layer $\left(b_{i}\right)$. Furthermore, a scale factor applied to the output weights $\left(w_{\mathrm{nc}}^{\prime}=k w_{\mathrm{nc}}\right.$, for $n=0, \ldots, N$ and $c=1, \ldots, C$ ) does not affect the final performance of the network, since if $b_{i}>b_{j}$, then $k b_{i}>k b_{j}$. This property allows scaling the output weights so that the maximum value of $w_{\mathrm{nc}}$ uses the entire dynamic range, minimizing the effects of the limited precision over the quantization of the output weights.

In this paper, all the experiments have been carried out using the MATLAB's Neural Network Toolbox [15], and the MLPs have been trained using the Levenberg-Marquardt algorithm with Bayesian regularization. The main advantage of using regularization techniques is that the generalization capabilities of the classifier are improved, and that it is possible to obtain better results with smaller networks, since the regularization algorithm itself prunes those neurons that are not strictly necessary.

\section{Definition of the Problem}

As mentioned in the introduction, there are two different (although strongly linked) topics that play a key role in the performance of the NN-based sound classifier, and that constitute the core of this paper. The first one, the quantization of the NN weights, will be described in Section 3.1, while the second issue, the feasibility of simplifying the NN activation function, will be stated in Section 3.2.

3.1. The Quantization Problem. Most of the actual DSPs for hearing aids make use of a 16-bit word-length Harvard Architecture, and only modern hearing instruments have larger internal bit range for number presentation (22-24 bits). In some cases, the use of larger numerical representations is reserved for the filterbank analysis and synthesis stages, or to the Multiplier/ACcumulator (MAC) that multiplies 16-bit registers, and stores the result in a 40-bit accumulator. In this paper we have focused on this last case, in which we have 


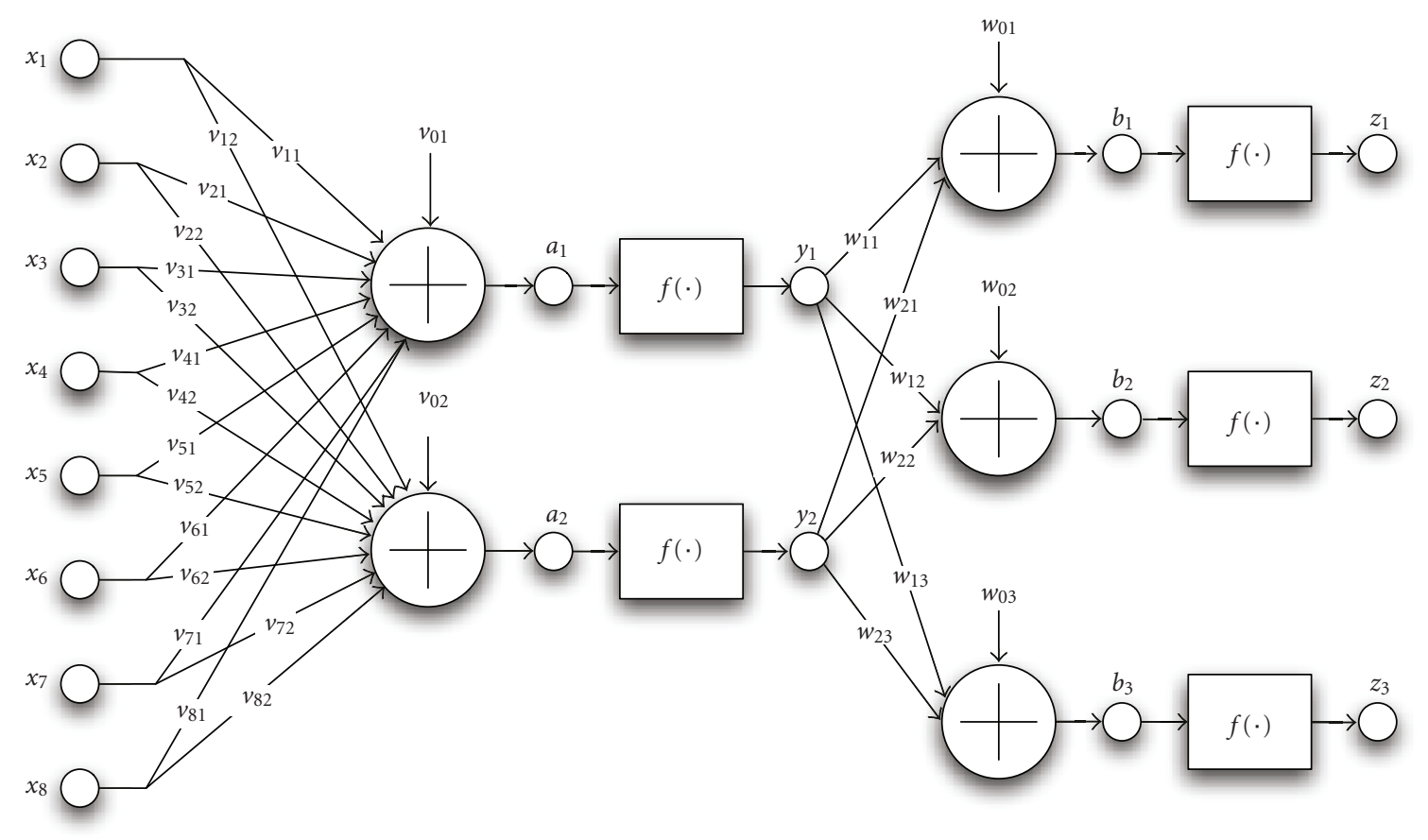

Figure 1: Multilayer Perceptron (MLP) diagram.

thus 16-bit to represent numbers, and, as a consequence, there are several 16-bit fixed-point quantization formats. It is important to highlight that in those modern DSPs that use larger numerical representations the quantization problem is minimized, since there are several configurations that yield very good results. The purpose of our study is to demonstrate that a 16 bit numerical representation configured in a proper way can produce considerably good results in the implementation of a neural classifier.

The way numbers are represented on a DSP is of crucial importance. Fixed-point numbers are usually represented by using the so-called " $Q$ number format." Within the application at hand, the notation more commonly used is "Qx.y", where

(i) $Q$ labels that the signed fixed-point number is in the "Q format notation,"

(ii) $x$ symbolizes the number of bits used to represent the 2's complement of the integer portion of the number,

(iii) $y$ designates the number of bits used to represent the 2 's complement of the fractional part of such number.

For example, using a numerical representation of 16 bits, we could decide to use the quantization Q16.0, which is used for representing 16-bit 2's complement integers. Or we could use Q8.8 quantization, what, in turns, means that 8 bits are used to represent the 2's complement of the integer part of the number, and 8 bits are used to represent the 2's complement of the fractional portion; or Q4.12, which assigns 4 bits to the integer part, and 12 bits to the fractional portion and so forth. The question arising here is: What is the most adequate quantization configuration for the hearing aid performance?
Apart from this question to be answered later on, there is also a crucial problem related to the small number of bits available to represent the integer and the fractional parts of numbers: the limited precision. Although not clear at first glance, it is worth noting that a low number of bits for the integer part may cause the register to saturate, while a low number of bits in the fractional portion may cause a loss of precision in the number representation.

3.2. The Problem of Approximating the Activation Function. As previously mentioned, the activation function in our NN is the hyperbolic tangent function which, in order to be implemented on a DSP, requires a proper approximation. To what extent an approximation $\tilde{f}$ is adequate enough is a balance between how well it "fits" $f$ and the number of instructions the DSP requires to compute $\tilde{f}$.

In the effort of finding a suitable enough approximation, in this work we have explored 2 different approximations for the hyperbolic tangent function, $f$. In general, the way an approximation, $\tilde{f}(x, \phi)$, fits $f$ will depend on a design parameter, $\phi$, whose optimum value has to be computed by minimizing some kind of error function. In this paper we have decided to minimize the root mean square error (RMSE) for input values uniformly distributed from -5 to +5 :

$$
\operatorname{RMSE}(f, \tilde{f})=+\sqrt{\hat{E}\left\{(f(x)-\tilde{f}(x))^{2}\right\}}
$$

The first practical implementation for approximating $f(x)$ is, with some corrections that will be explained below, based on a table containing the main $2^{n}=256$ values of $f(x)=\tanh (x)$. Such approximation, which makes use of 
256 tabulated values, has been labeled $f_{T 256}(x)$, and, for reasons that will be explained below, has been defined as

$$
f_{T 256}(x)= \begin{cases}+1, & x>2^{n-1-b}, \\ \tanh \left(\left\lfloor x \cdot 2^{b}\right\rfloor 2^{-b}\right), & 2^{n-1-b} \geq x \geq-2^{n-1-b}, \\ -1, & x<-2^{n-1-b}\end{cases}
$$

with $b$ being a design parameter to be optimized by minimizing its root mean square error $\operatorname{RMSE}\left(f, f_{T 256}\right)$, making use of the proper particularization of Expression (7).

The "structure" that $f_{\text {T256 }}$ approximation exhibits in (8) requires some comments.

(1) Expression (8) assigns +1 output to those input values greater than $2^{n-1-b}$, and -1 output to those input values lower than $-2^{n-1-b}$. With respect to the remaining input values belonging to the interval $2^{n-1-b} \geq x \geq-2^{n-1-b}, f_{T 256}$ divides such interval into $2^{n}$ possible values, whose corresponding output values have been tabulated and stored in RAM memory.

(2) We have included in (8), for reasons that will appear clearer later on, the scale factor $2^{b}$, aiming at determining which are the bits of $x$ that lead to the best approximation of function $f$.

(3) The $b$ parameter in the aforementioned scale factor determines the way $f_{T 256}$ approaches $f$. Its optimum value is the one that minimizes the root mean square error $\operatorname{RMSE}\left(f, f_{T 256}\right)$. In this respect, Figure 2 represents the $\operatorname{RMSE}\left(f, f_{T 256}\right)$ as a function of the $b$ parameter, and shows that the minimum value of RMSE $\left(\mathrm{RMSE}_{\min }=0.0025\right)$ is obtained when $b=$ $b_{\text {opt }}=5.4$.

(4) Since, for practical implementation, $n$ is an integer number, we take $b=5$ as the closest integer to $b_{\mathrm{opt}}=$ 5.4. This leads to $\mathrm{RMSE}=0.0035$.

(5) The scale factor $2^{5}$ in Expression (8) (multiplying by $2^{5}$ ) is equivalent to binary shift $x$ in 5 bits to the left, which can be implemented using only one assembler instruction!

As a consequence, implementing the $f_{T 256}$ approximation requires storing 256 memory words, and the following 6 assembler instructions:

(1) shifting 5 bits to the left,

(2) a saturation operation,

(3) a 8-bit right shift,

(4) the addition of the starting point of the table in memory,

(5) copying this value to an addressing register,

(6) reading the value in the table.

However, in some cases (basically, when the number of neurons is high), this number of instructions is too long.

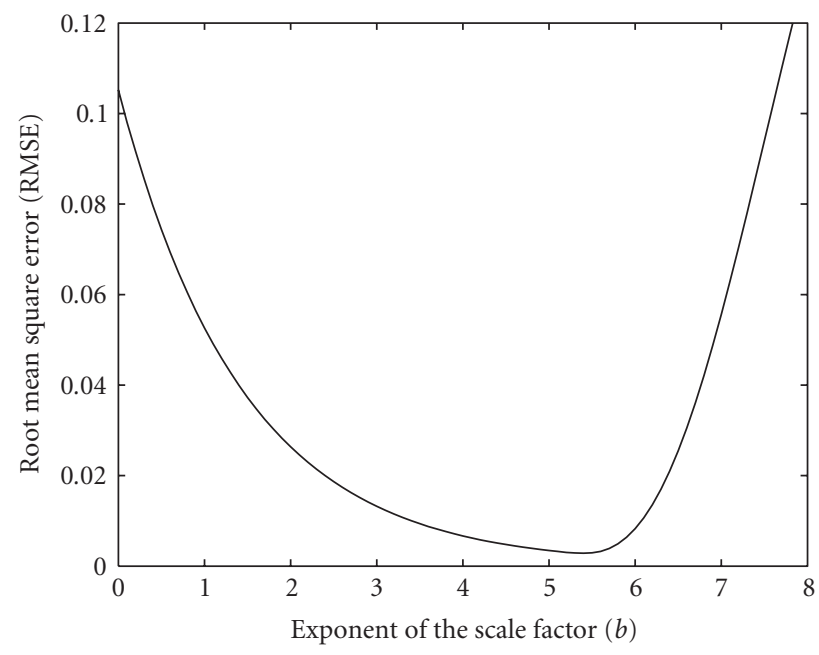

Figure 2: $\operatorname{RMSE}\left(f, f_{T 256}\right)$, root mean square error of the tabulatedbase approximation, as a function of the $b$ parameter, the exponent of the scale factor in its defining Expression (8).

In order to simplify the calculation of this approximated function, or in other words, to reduce the number of instructions, we have tested a second approach based on a piecewise approximation. Taking into account that a typical DSP is able to implement a saturation using one cycle, we have evaluated the feasibility of fitting the original activation function $f$ by using a function, which is based on 3-piece linear approximation, has been labelled ( $\left.f_{3 \text { PLA }}\right)$, and exhibits the expression:

$$
f_{3 \mathrm{PLA}}(x)= \begin{cases}1, & x>\frac{1}{a}, \\ a \cdot x, & \frac{1}{a} \geq x \geq-\frac{1}{a}, \\ -1, & x<-\frac{1}{a},\end{cases}
$$

where subscript "3PLA" stands for "3-piece linear approximation," and $a$ is the corresponding design parameter, whose optimum value is the one that optimizes the $\operatorname{RMSE}\left(f, f_{3 \text { PLA }}\right)$. Regarding this optimization process, Figure 3 shows the $\operatorname{RMSE}\left(f, f_{3 \mathrm{PLA}}\right)$ as a function of the $a$ parameter. Note that the $a$ value that makes the $\operatorname{RMSE}\left(f, f_{3 \mathrm{PLA}}\right)$ be minimum $(0.0445)$ is $a_{\text {opt }}=0.769$.

The practical point to note here regarding this approximation is that it requires multiplying the input of the activation function by $a$, that, in a typical DSP requires, at least, the following 4 instructions:

(1) copying $x$ into one of the input register of the MAC unit,

(2) copying the constant value of $a$ into the other input register,

(3) copying the result of this multiplication into the accumulator,

(4) a saturation operation.

As a consequence, the minimum number of instructions required a priori for implementing this approximation is 


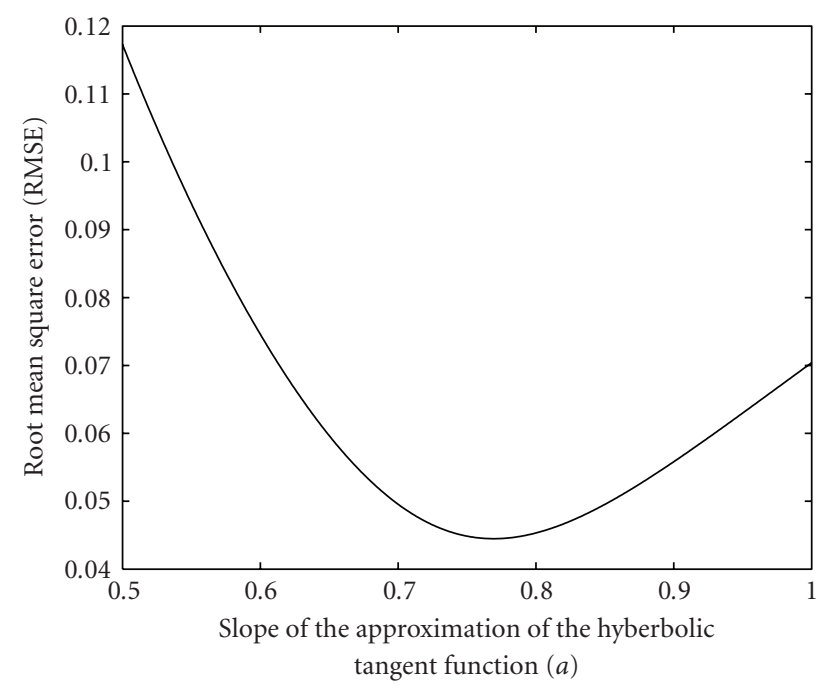

FIgURe 3: Root mean square corresponding to the 3-piece linear approximation, $\operatorname{RMSE}\left(f, f_{3 \mathrm{PLA}}\right)$, as a function of the a parameter, the slope in its defining Expression (9).

4, since the saturation operation requires an additional assembler instruction.

Furthermore, a possible way of reducing even more the number of instructions required for implementing an approximation consists in including the term $a$ in the corresponding weights of the neuron, so that $f_{3 \mathrm{PLA}}(x, a=$ $0.769)=f_{3 \text { PLA }}(0.769 x, a=1)$. So, the additional bonus achieved consists in that the number of instructions is drastically reduced to only 1 assembler instruction.

For illustrative purposes, we complete this Section by having a look at Figure 4. It represents the 2 approximations considered in the paper: the "tabulated function-based" function $\left(f_{T 256}\right.$, with $\left.b=5\right)$ and the 3-piece linear approximation with $\left(f_{3 \text { PLA }}\right.$, with $\left.a=0.769\right)$.

\section{Experimental Work}

Prior to the description of the different experiments we have carried out, it is worth having a look at the sound database we have used. It consists of a total of 7340 seconds of audio, including both speech in quiet, speech in noise, speech in music, vocal music, instrumental music and noise. The database was manually labelled, obtaining a total of 1272.5 seconds of speech in quiet, 3637.5 seconds of speech in music or noise and 2430 seconds of music and noise. All audio files are monophonic, and were sampled with a sampling frequency of $16 \mathrm{kHz}$ and 16 bits per sample. Speech and music files were provided by D. Ellis, and recorded by E. Scheirer and M. Slaney [16]. This database [17] has already been used in a number of different works [16, 18-20]. Speech was recorded by digitally sampling FM radio stations, using a variety of stations, content styles and levels, and contains samples from both male and female speakers. The sound files present different input levels, with a range of $30 \mathrm{~dB}$ between the lowest and the highest, which allows us to test the

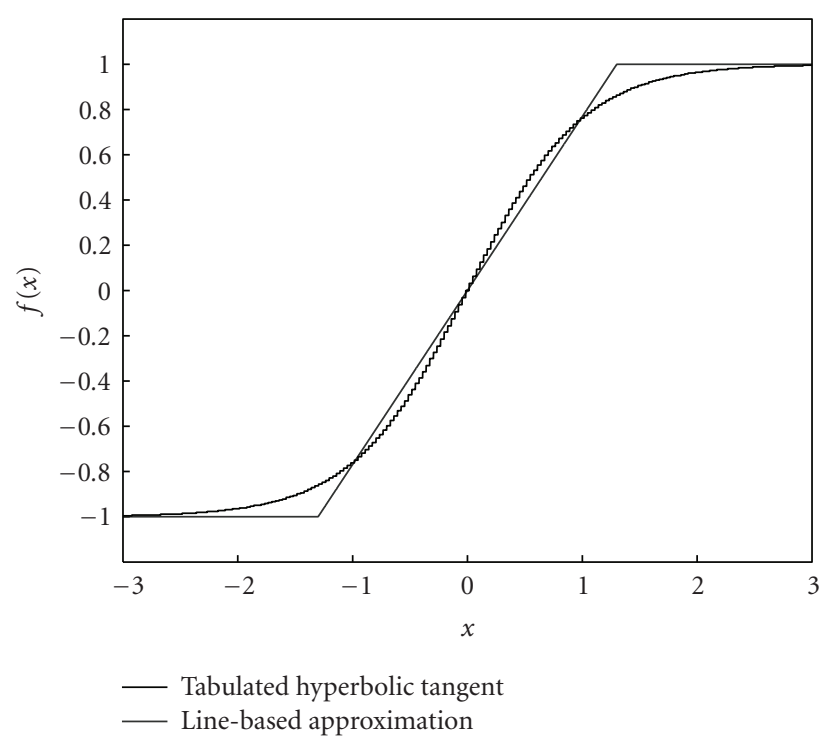

FIGURE 4: Representation of the considered activation functions. tabulated hyperbolic function and line-based approximation.

robustness of the classification system against different sound input levels. Music includes samples of jazz, pop, country, salsa, reggae, classical, various nonWestern styles, various sorts of rock, and new age music, both with and without vocals. Finally, noise files include sounds from the following environments: aircraft, bus, cafe, car, kindergarten, living room, nature, school, shop, sports, traffic, train, and train station. These noise sources have been artificially mixed with those of speech files (with varying degrees of reverberation) at different Signal to Noise Ratios (SNRs) ranging from 0 to $10 \mathrm{~dB}$. In a number of experiments, these values have been found to be representative enough regarding the following perceptual criteria: lower SNRs could be treated by the hearing aid as noise, and higher SNRs could be considered as clean speech.

For training, validation, and testing, it is necessary for the database to be divided into three different sets. 2685 seconds $(\approx 36 \%)$ for training, 1012.5 seconds $(\approx 14 \%)$ for validation, and 3642.5 seconds $(\approx 50 \%)$ for testing. This division has been done randomly, ensuring that the relative proportion of files of each category is preserved for each set. The training set is used to determine the weights of the MLP in the training process, the validation set helps evaluate progress during training and to determine when to stop training, and the test set is used to assess the classifier's quality after training. The test set has remained unaltered for all the experiments described in this paper.

Each file was processed using the hearing aid simulator described in [21] without feedback. The features were computed from the output of the Weighted Overlap-Add (WOLA) filterbank with 128 DFT points and analysis and synthesis window lengths of 256 samples. So, the time/frequency decomposition is performed with 64 frequency bands. Concerning the architecture, the simulator has been configured for a 16-bit word-length Harvard 
TABLE 1: Mean error probability (\%) of different classifiers returning a decision with time slots of 2.5 seconds using 9 quantization schemes: $Q x \cdot y$ represents the quantization schemes with $x$ bits for the integer part, and $y$ for the fractional one. Regarding the classifiers,MLP $K$ means Multi-Layer Perceptron with $K$ neurons in the hidden layer. The column labelled "Double" corresponds to the Mean error probability (\%) when no-quantization, double floating point precision has been used. Columns in bold aim at helping the reader focus on the most relevant result: Q5.11 provides very similar results to those of double precision.

\begin{tabular}{lcccccccccc}
\hline & No quantization & \multicolumn{9}{c}{ Quantization scheme } \\
Classifier & Double & Q1.15 & Q2.14 & Q3.13 & Q4.12 & Q5.11 & Q6.10 & Q7.9 & Q8.8 & Q9.7 \\
\hline MLP 1 & $\mathbf{1 5 . 1 5}$ & 55.63 & 55.30 & 20.94 & 15.16 & $\mathbf{1 5 . 2 1}$ & 15.30 & 15.79 & 23.33 & 36.28 \\
MLP 2 & $\mathbf{1 0 . 4 6}$ & 73.43 & 37.46 & 15.76 & 10.47 & $\mathbf{1 0 . 4 7}$ & 10.48 & 10.88 & 15.55 & 36.63 \\
MLP 3 & $\mathbf{9 . 8 4}$ & 71.90 & 38.16 & 12.25 & 9.88 & $\mathbf{9 . 8 5}$ & 9.86 & 10.21 & 16.76 & 44.69 \\
MLP 4 & $\mathbf{9 . 1 6}$ & 74.60 & 42.41 & 14.04 & 9.26 & $\mathbf{9 . 1 7}$ & 9.20 & 9.67 & 16.95 & 46.71 \\
MLP 5 & $\mathbf{8 . 8 6}$ & 69.08 & 42.11 & 13.76 & 8.92 & $\mathbf{8 . 8 6}$ & 8.92 & 9.58 & 17.75 & 40.56 \\
MLP 6 & $\mathbf{8 . 5 5}$ & 65.08 & 35.32 & 11.07 & 8.58 & $\mathbf{8 . 5 4}$ & 8.58 & 9.27 & 17.13 & 41.99 \\
MLP 7 & $\mathbf{8 . 3 9}$ & 65.91 & 38.18 & 10.57 & 8.40 & $\mathbf{8 . 4 0}$ & 8.46 & 9.41 & 18.84 & 42.45 \\
MLP 8 & $\mathbf{8 . 3 3}$ & 62.37 & 33.43 & 9.51 & 8.33 & $\mathbf{8 . 3 4}$ & 8.41 & 8.98 & 17.31 & 44.01 \\
MLP 9 & $\mathbf{8 . 3 4}$ & 61.17 & 34.76 & 10.45 & 8.53 & $\mathbf{8 . 3 4}$ & 8.35 & 9.11 & 17.76 & 43.88 \\
MLP 10 & $\mathbf{8 . 1 7}$ & 62.19 & 34.27 & 9.30 & 8.18 & $\mathbf{8 . 1 9}$ & 8.26 & 8.96 & 17.76 & 43.06 \\
MLP 15 & $\mathbf{8 . 1 0}$ & 62.03 & 32.79 & 9.22 & 8.11 & $\mathbf{8 . 1 1}$ & 8.18 & 8.96 & 17.36 & 40.41 \\
MLP 20 & $\mathbf{7 . 9 3}$ & 51.67 & 29.03 & 9.42 & 7.92 & $\mathbf{7 . 9 2}$ & 7.97 & 8.85 & 18.17 & 44.11 \\
MLP 25 & $\mathbf{7 . 9 4}$ & 61.27 & 32.75 & 9.91 & 7.94 & $\mathbf{7 . 9 4}$ & 8.01 & 8.98 & 17.96 & 41.69 \\
MLP 30 & $\mathbf{7 . 8 6}$ & 59.31 & 35.45 & 10.13 & 7.92 & $\mathbf{7 . 8 7}$ & 7.91 & 8.73 & 17.46 & 42.52 \\
MLP 35 & $\mathbf{7 . 9 5}$ & 59.84 & 32.12 & 10.02 & 7.99 & $\mathbf{7 . 9 5}$ & 8.01 & 8.85 & 17.81 & 43.47 \\
MLP 40 & $\mathbf{7 . 7 8}$ & 59.71 & 30.78 & 10.15 & 7.77 & $\mathbf{7 . 7 4}$ & 7.82 & 8.74 & 17.72 & 41.27 \\
\hline
\end{tabular}

Architecture with a Multiplier/ACcumulator (MAC) that multiplies 16-bit registers and stores the result in a 40-bit accumulator.

In order to study the effects of the limited precision, two different scenarios were considered in the experiments. First, the classifiers were configured for returning a decision every 2.5 seconds. The aim of this study is to determine the effects of the limited precision over the classifiers for applications like automatic program switching, in which a large time scale is used. Second, the classifiers were configured for taking a decision with time slots of 20 milliseconds. In this case, the objective is to study the effects of the limited precision in a classification scenario in which a small time scale is required like, for example, in noise reduction or sound source separation applications.

In the batches of experiments we have put into practice, the experiments have been repeated 100 times. The results we have illustrated below show the average probability of classification error for the test set and the computational complexity in number of assembler operations needed to obtain the output of the classifier. The probability of classification error represents the average number of time slots that are misclassified in the test set.

It is important to highlight that in a real classification system the classification evidence can be accumulated across the time for achieving lower error rates. This fact makes necessary a study of the tradeoff between the selected time scale, the integration of decision for consecutive time slots, the performance of the final system and the required computational complexity. This analysis is out of the scope of this paper, since our aim is not to propose a particular classification system, that must be tuned for the considered hearing aid application, but to illustrate a set of tools and strategies that can be used for determining the way a neural network can be efficiently implemented in real time for sound environment classification tasks with limited computational capabilities.

4.1. Comparing the Quantization Schemes. The objective of this first set of experiments is to study the effects of the quantization format, Qx.y, used for representing both the signal describing features and the weights of the neural network. In this experimental work, aiming at clearly distinguishing the different phenomena involved, the activation function used in the neural network is the original hyperbolic tangent function, $f$. The influence of using some of the aforementioned approximation of $f$ has also been explored in a novel sequence of experiments whose results will be explained in Section 4.2.

Tables 1 and 2 show the average probability of error (\%) obtained in the 100 runs of the training process for a variety of multilayer perceptrons (MLPs) with different numbers of hidden neurons, for time slots of 2.5 seconds and 20 milliseconds, respectively. In these tables, MLP $K$ labels that the corresponding NN is an MLP with $K$ neurons in the hidden layer. These batches of experiments have explored a numbers of hidden neurons ranging from 1 to 40. Aiming at clearly understanding the effect of the different quantization schemes, we have also listed the average probability of error computed with no-quantization, double floating point precision. These have been labeled in Tables 1 and 2 by using the header "double." 
TABLe 2: Mean error probability (\%) of different classifiers returning a decision with time slots of 20 milliseconds using 9 quantization schemes: $Q x \cdot y$ represents the quantization schemes with $x$ bits for the integer part, and $y$ for the fractional one. Regarding the classifiers, MLP $K$ means Multi-Layer Perceptron with $K$ neurons in the hidden layer. The column labelled "Double" corresponds to the Mean error probability (\%) when no-quantization, double floating point precision has been used. Columns in bold aim at helping the reader focus on the most relevant result: Q5.11 provides very similar results to those of double precision.

\begin{tabular}{lcccccccccc}
\hline & No quantization & \multicolumn{9}{c}{ Quantization scheme } \\
Classifier & Double & Q1.15 & Q2.14 & Q3.13 & Q4.12 & Q5.11 & Q6.10 & Q7.9 & Q8.8 & Q9.7 \\
\hline MLP 1 & $\mathbf{3 6 . 3 6}$ & 44.05 & 41.25 & 37.24 & 36.36 & $\mathbf{3 6 . 3 6}$ & 36.42 & 37.11 & 41.79 & 60.16 \\
MLP 2 & $\mathbf{2 7 . 4 4}$ & 42.88 & 33.10 & 28.28 & 27.45 & $\mathbf{2 7 . 4 6}$ & 27.88 & 32.21 & 46.19 & 60.96 \\
MLP 3 & $\mathbf{2 6 . 1 1}$ & 45.86 & 44.42 & 37.05 & 31.23 & $\mathbf{2 6 . 6 0}$ & 27.43 & 36.97 & 49.26 & 61.56 \\
MLP 4 & $\mathbf{2 4 . 6 1}$ & 50.66 & 51.47 & 41.38 & 30.18 & $\mathbf{2 4 . 9 3}$ & 26.52 & 36.60 & 54.79 & 62.17 \\
MLP 5 & $\mathbf{2 3 . 0 7}$ & 50.91 & 46.39 & 39.42 & 28.25 & $\mathbf{2 3 . 4 5}$ & 27.07 & 41.88 & 57.32 & 65.41 \\
MLP 6 & $\mathbf{2 2 . 1 8}$ & 55.34 & 51.77 & 45.29 & 30.43 & $\mathbf{2 3 . 4 3}$ & 27.17 & 39.41 & 54.45 & 62.82 \\
MLP 7 & $\mathbf{2 1 . 5 0}$ & 53.69 & 49.61 & 44.22 & 28.74 & $\mathbf{2 2 . 3 5}$ & 26.53 & 39.00 & 54.37 & 63.40 \\
MLP 8 & $\mathbf{2 1 . 0 7}$ & 54.80 & 52.90 & 47.81 & 26.42 & $\mathbf{2 1 . 9 5}$ & 25.54 & 36.53 & 53.47 & 61.41 \\
MLP 9 & $\mathbf{2 0 . 5 5}$ & 56.32 & 50.24 & 47.41 & 26.81 & $\mathbf{2 1 . 7 5}$ & 23.44 & 36.77 & 53.16 & 60.83 \\
MLP 10 & $\mathbf{2 0 . 8 0}$ & 58.96 & 52.28 & 49.60 & 28.18 & $\mathbf{2 2 . 3 0}$ & 23.71 & 36.65 & 52.84 & 61.20 \\
MLP 15 & $\mathbf{1 9 . 7 4}$ & 61.13 & 56.33 & 52.93 & 30.14 & $\mathbf{2 0 . 8 3}$ & 21.48 & 32.83 & 51.28 & 63.11 \\
MLP 20 & $\mathbf{1 9 . 5 4}$ & 62.85 & 57.45 & 53.50 & 29.36 & $\mathbf{2 0 . 1 9}$ & 20.94 & 30.47 & 49.57 & 61.71 \\
MLP 25 & $\mathbf{1 9 . 4 9}$ & 62.54 & 57.30 & 53.40 & 30.97 & $\mathbf{2 0 . 3 6}$ & 20.90 & 30.20 & 49.88 & 63.60 \\
MLP 30 & $\mathbf{1 9 . 4 7}$ & 63.99 & 57.14 & 51.93 & 31.53 & $\mathbf{2 0 . 2 5}$ & 20.61 & 28.93 & 48.82 & 61.23 \\
MLP 35 & $\mathbf{1 9 . 4 4}$ & 64.87 & 56.70 & 52.14 & 32.19 & $\mathbf{2 0 . 9 4}$ & 20.41 & 26.69 & 45.07 & 60.02 \\
MLP 40 & $\mathbf{1 9 . 4 9}$ & 62.67 & 55.06 & 49.96 & 29.78 & $\mathbf{2 0 . 2 9}$ & 20.37 & 27.67 & 46.32 & 61.19 \\
\hline
\end{tabular}

Tables 1 and 2 supply some important pieces of useful information:

(i) Those quantization formats with a low number of bits for representing the integer part, such as, for example, Q2.14, finally lead to an increase in the error probability when compared to those computed with double precision. This increase is caused by saturations of the features and weights of the neural networks.

(ii) On the other hand, the use of a low number of bits for the fractional portion causes an increase in the error probability, basically arising from the loss of precision in the numerical representation.

These facts illustrate the need for a tradeoff between integer and fractional bits. For the sake of clarity, Figure 5 shows the average relative increase in the error probability with respect to the use of double precision, as a function of the number of bits of the fractional portion. Computing this relative increase has required the use of those results obtained when using all the classifiers listed in Tables 1 and 2, and the average computed from

$$
I=100 \cdot \hat{E}\left\{\frac{P_{Q i . j}-P_{\text {double }}}{P_{\text {double }}}\right\}(\%),
$$

where $\hat{E}\{\cdot\}$ represents the mean value of the probabilities involving all the number of neurons considered. Note that the lower relative increase is achieved by the Q5.11 quantization scheme, for both time slot configurations. This

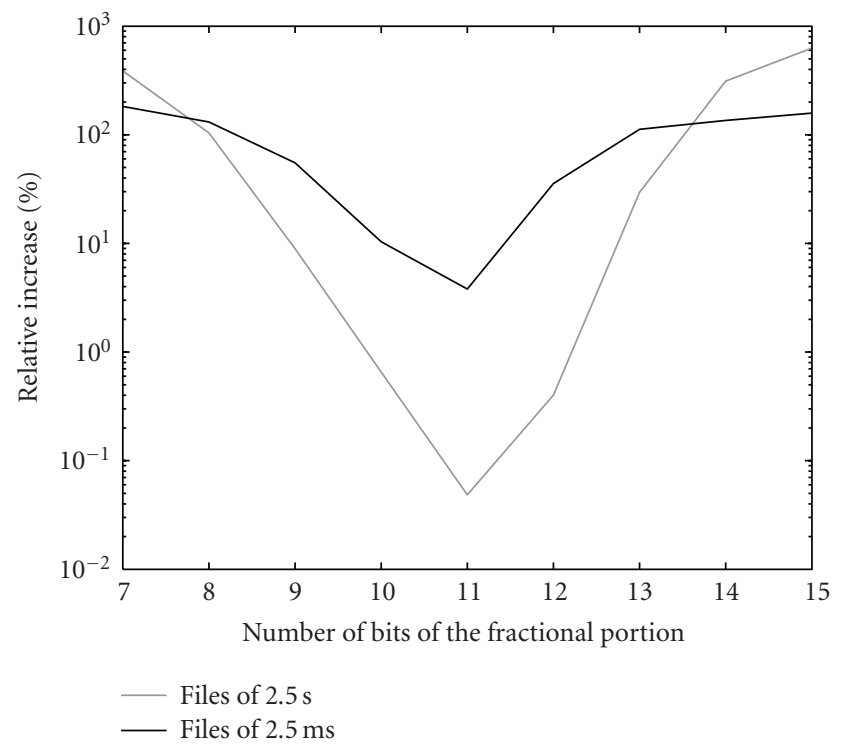

FIGURE 5: Average relative increase (\%) in the probability of error for the classifiers studied in this paper.

is the reason by which the Q5.11 quantization format has been selected for the remaining experiments of the paper.

4.2. Comparing the Approximation of the Activation Functions. The purpose of this second batch of experiments consists in quantitatively evaluating the fitness of the approximations 
TABLE 3: Mean error probability (\%) and number of simple operations required for computing the activation function approximations when using neural networks with different activation functions: the "tabulated function-based" function $\left(f_{T 256}\right.$, with $\left.b=5\right)$ and the 3-piece linear approximation with $\left(f_{3 P L A}\right.$, with $\left.a=0.769\right)$. MLP X means that the multilayer perceptron under study contains X hidden neurons.

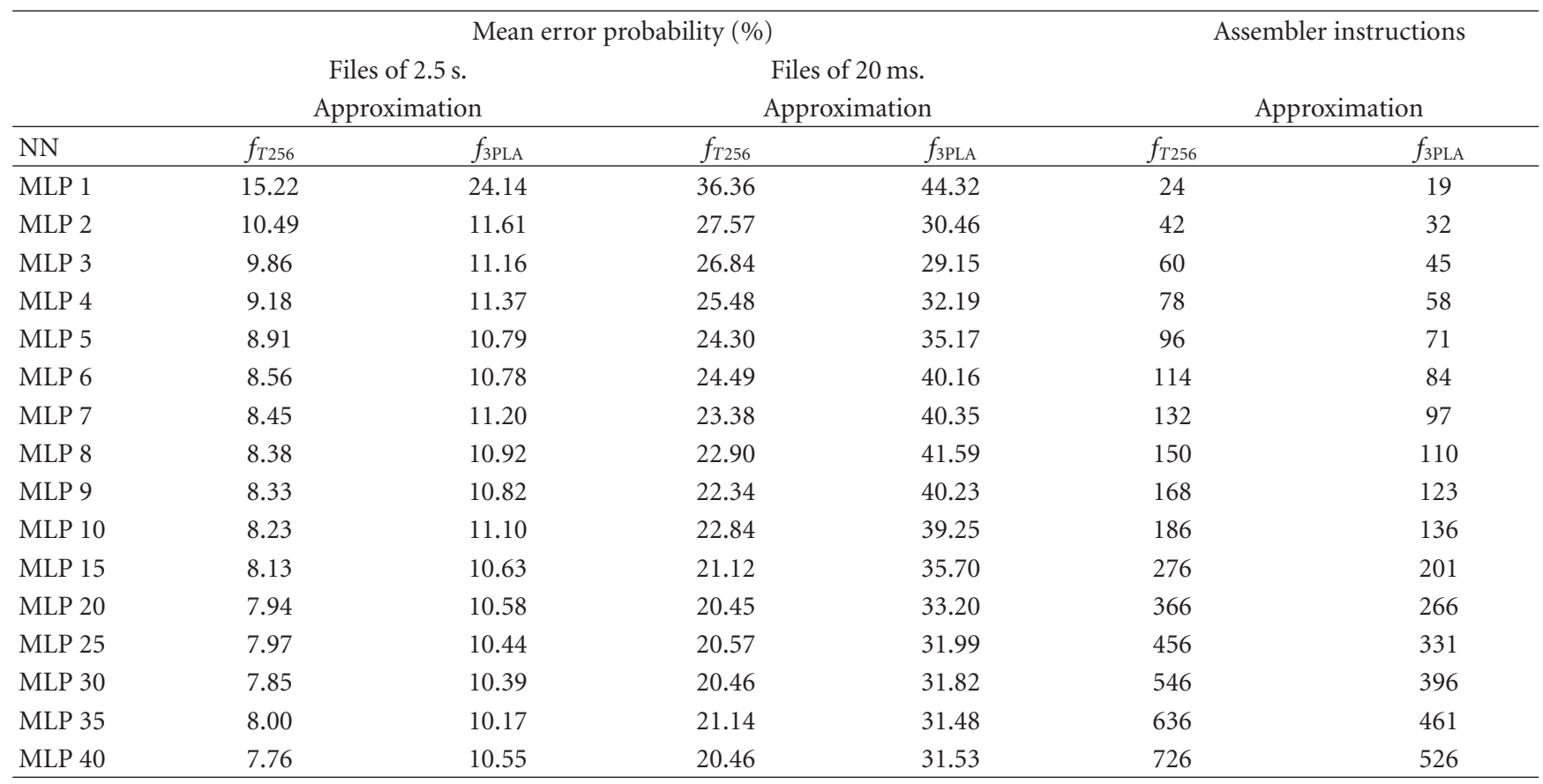

explored in the paper: the "tabulated function-based" function $\left(f_{T 256}\right.$, with $\left.b=5\right)$ and the 3-piece linear approximation with ( $f_{3 \text { PLA }}$, with $\left.a=0.769\right)$. The quantization scheme we have used in this experimental work is Q5.11 because, as stated in Section 4.1, it is the one that makes the different classifiers achieve very similar results as those obtained when no quantization (double precision) is used.

Table 3 shows the error probability corresponding to MLPs (ranging from 1 to 40 hidden neurons) that make use of the aforementioned approximations, for files of 2.5 seconds and 20 milliseconds, respectively. A detailed observation of Table 3 leads to the following conclusions.

(i) The "tabulated function-based" approximation, $f_{T 256}$, makes the NNs achieve very similar results to those obtained when using the original hyperbolic tangent function, $f$, for the case of files of 2.5 seconds (average relative increase of $0.30 \%$ ), and an average relative increase of $5.91 \%$, for the case of files of 20 milliseconds. The way to note this consists in comparing the mean error probabilities listed on column Q5.11 in Tables 1 and 2 (in which the activation function has not yet been approximated) with those corresponding to columns " $f_{T 256}$ " in Table 3.

(ii) The use of the 3-piece linear approximation, $f_{3 \text { PLA, }}$, leads to an average relative increase in the probability of error of $29.88 \%$ and $61.27 \%$ for files of 2.5 seconds and 20 milliseconds, respectively.

As a conclusion, we can say that the "tabulated functionbased" approximation, $f_{T 256}$, is a suitable way to approach the original hyperbolic tangent function, $f$, mainly for the case of files of 2.5 seconds.

Another extremely important point to note is that both the considered approximations for the activation function and the number of neurons are related to the number of assembler instructions needed to implement the classification system in the hearing aid. In this respect, Table 3 also shows the number of instructions for the different MLP $K$ classifiers ( $K$ being the numbers of hidden neurons) as a function of the approximation for the hyperbolic tangent function $\left(f_{T 256}\right.$ and $f_{3 \text { PLA }}$ ).

4.3. Improving the Results by Retraining the Output Weights. As can be shown from the results obtained in the previous section, the use of approximated activation functions reduces the number of assembler instructions needed to implement the classifier. Even though this is a positive fact, the use of approximation for the activation functions may cause the classifier to slightly reduce its efficiency. Aiming at overcoming this, we have carried out a novel sequence of experiments, which consists in what follows.

(1) Train the NN.

(2) Introduce the aforementioned quantization schemes and the approximations for the activation function.

(3) Recompute the output weights of the network by taking into account the studied effects related to quantization schemes and the approximations for the activation function.

Note that training the MLP directly with the quantization schemes and the approximations for the activation function 
TABLE 4: Mean error probability (\%) and number of simple operations required for computing the activation function approximations when using neural networks with different activation functions, when the output weights are retrained once the activation function is applied.

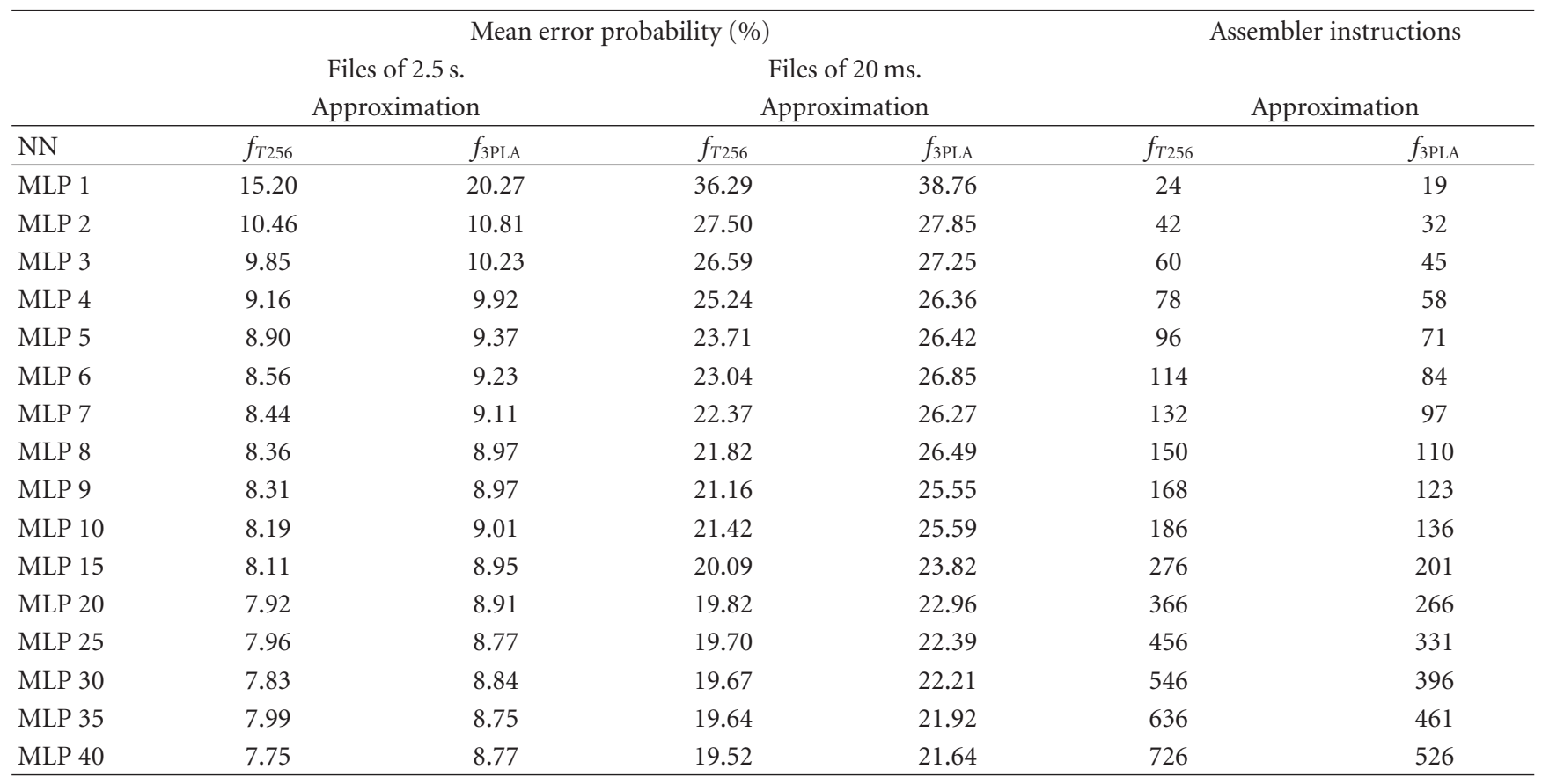

is not straightforward since the approximations used for the activation functions are not differentiable at some points, or their slope is zero. The solution proposed here overcomes these problems, and makes the process much easier.

Table 4 shows the mean error probability obtained by the different neural networks once the output weights have been recomputed. Understanding Table 4 requires to compare it to Table 3 (in which these have not been recalculated). From this comparison, we would like to emphasize the following.

(i) The retrained strategy slightly reduces the error when the tabulated-approximation is used. Now, $f_{T 256}$ leads to an average relative increase in the probability of error of $0.13 \%$ and $1.94 \%$ for files of 2.5 second and 20 millisecond, respectively, compared to those obtained when no quantization (double precision) is used.

(ii) In the case of the 3-piece-based approximation, the retrained strategy leads to an average relative increase in the probability of error of $10.36 \%$ and $15.08 \%$ for files of $2.5 \mathrm{~s}$. and $20 \mathrm{~ms}$., respectively, compared to those obtained when double precision is used.

To complete this paper, and in order to compare the benefits of the proposed retraining strategy with those results presented in the previous section, Figures 6 and 7 show the relationship between the error rate and the number of operations for the tabulated-based implementation and for the line-based implementation with and without retrained output weights, for files of 2.5 seconds and 20 milliseconds, respectively. Taking into account the limited

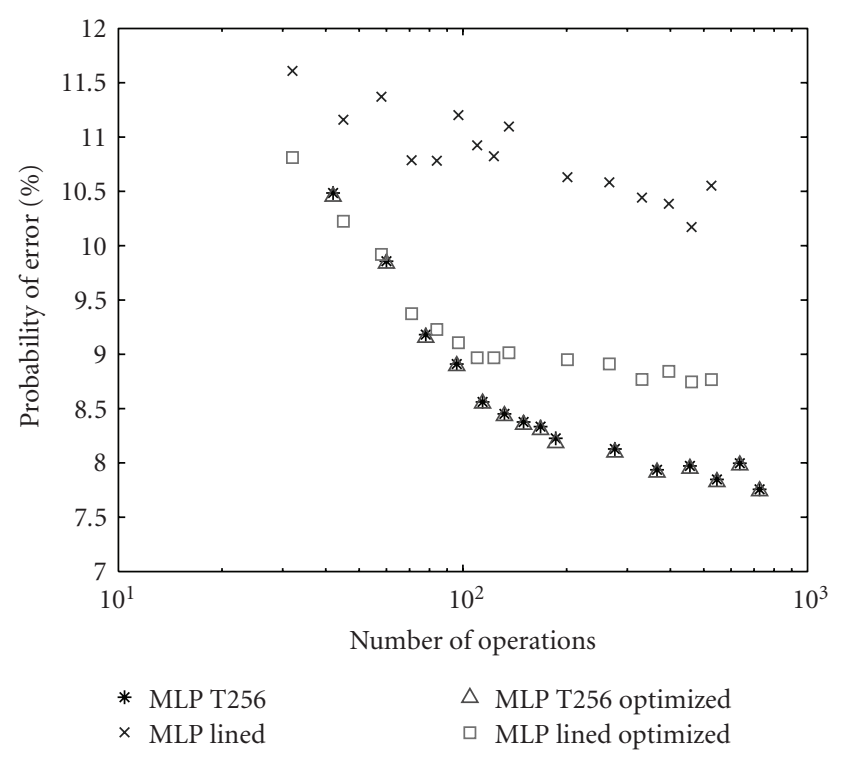

FIGURE 6: Comparative analysis of the relationship between the error rate and the number of operations for the best methods studied in the paper.

number of operations per second (low clock rates in order to minimize power consumption), the results in Figures 6 and 7 demonstrate the effectiveness of the proposed strategy, especially in the case of time slots of 20 milliseconds, because it allows to achieve lower error rates with comparable computational complexity. Furthermore, the use of the linebased approximation is recommended mainly when very 


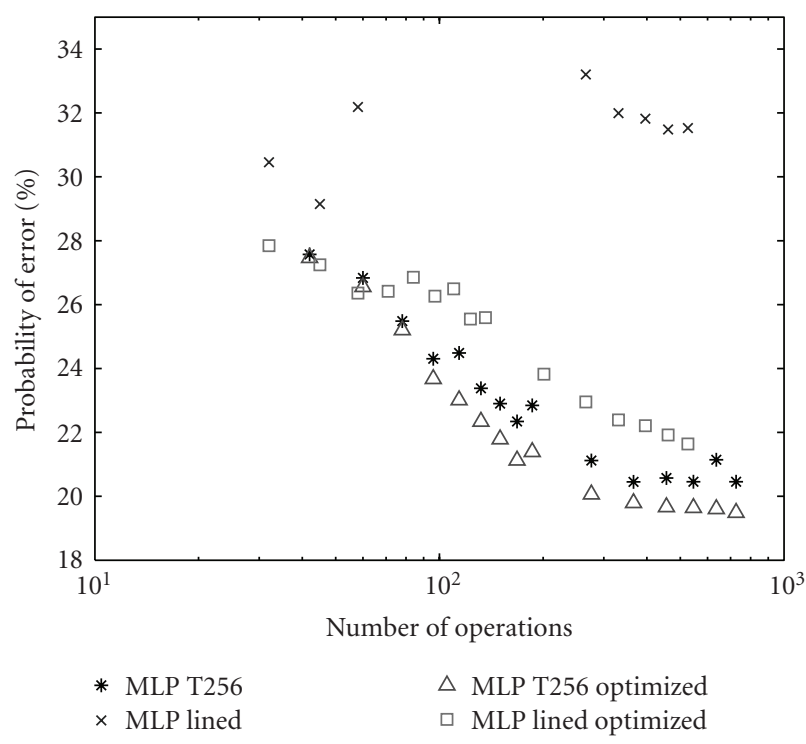

Figure 7: Comparative analysis of the relationship between the error rate and the number of operations for the best methods studied in the paper.

low computational complexity (less than 50 instructions) is required.

\section{Conclusions}

This paper has been motivated by the fact that the implementation of signal processing techniques on hearing aids is strongly constrained by (1) the finite precision used for representing numbers, and (2) the small number of instructions per second to implement the algorithms on the digital signal processor the hearing aid is based on. In this respect, the objective of this paper has been to quantitatively analyzing these effects on the performance of neural network-based sound classifiers in digital hearing aids. Such performance must be a delicate balance between keeping error classification probability within low values (in order to not disturb the user's comfort) and achieving this by using a small number of instructions per second. The reason underlying this latter restrictions is that hearing aids have to work at low clock rates in order to minimize the power consumption and maximize battery life.

Within this framework, the paper has particularly centered on exploring the following.

(1) the effects of using quantized weights and an approximated activation function for the neurons that compose the classifier. In particular, we have evaluated 2 different approximations; (1) the "tabulated function-based" function, based on 256 samples of the analytical activation function, and (2) the 3-piece linear approximation,

(2) how to improve the performance by making use of the information extracted from point 1.
The different batches of experiments lead to the following conclusions.

(i) The Q5.11 quantization scheme has been found to be exhibiting very similar results to those obtained when no-quantization, double-precision is used, mainly for the case of files of 2.5 seconds.

(ii) The "tabulated function-based" approximation makes the NNs achieve very similar results to those obtained when using the original hyperbolic tangent function for the case of files of 2.5 seconds, and an average relative increase of $5.91 \%$, for the case of files of 20 milliseconds.

(iii) The use of the 3-piece linear approximation leads to an average relative increase in the probability of error.

(iv) The retrained strategy reduces the error in average for all the experiments considered in the paper.

The final, global conclusion is that the quantized, approximated, neural network-based classifier achieves perceptually the same efficiency as that reached by "exact" networks (that is, without these approximations), but, and this is the key point, with the added bonus of extremely reducing the computational cost on the digital signal processor the hearing aid is based on.

\section{Acknowledgments}

This work has been partially funded by the Comunidad de Madrid/Universidad de Alcala (CCG06-UAH/TIC-0378, CCG07-UAH/TIC-1572, CCG08-UAH/TIC-4270) and the Spanish Ministry of Education and Science (TEC200613883-C04-04/TCM, TEC2009-14414-C03-03). The authors would like to thank the anonymous reviewers and the editor for their many helpful suggestions, which have greatly improved the presentation of this paper.

\section{References}

[1] V. Harnacher, J. Chalupper, J. Eggers, et al., "Signal processing in high-end hearing aids: state of the art, challenges, and future trends," EURASIP Journal on Applied Signal Processing, vol. 2005, no. 18, pp. 2915-2929, 2005.

[2] M. Büchler, S. Allegro, S. Launer, and N. Dillier, "Sound classification in hearing aids inspired by auditory scene analysis," EURASIP Journal on Applied Signal Processing, vol. 2005, no. 18, pp. 2991-3002, 2005.

[3] M. C. Büchler, Algorithms for sound classification in hearing instruments, Ph.D. thesis, Swiss Federal Institute of Technology, Zurich, Switzerland, 2002.

[4] M. Marzinzik, Noise reduction schemes for digital hearing aids and their use for hearing impaired, Ph.D. thesis, Carl von Ossietzky University, Oldenburg, Germany, 2000.

[5] J. Ramírez, J. C. Segura, C. Benítez, A. de la Torre, and A. Rubio, "Efficient voice activity detection algorithms using long-term speech information," Speech Communication, vol. 42, no. 3-4, pp. 271-287, 2004.

[6] J. M. Górriz, J. Ramírez, J. C. Segura, and C. G. Puntonet, "Improved MO-LRT VAD based on bispectra Gaussian model," Electronics Letters, vol. 41, no. 15, pp. 877-879, 2005. 
[7] P. Nordqvist and A. Leijon, "An efficient robust sound classification algorithm for hearing aids," Journal of the Acoustical Society of America, vol. 115, no. 6, pp. 3033-3041, 2004.

[8] E. Alexandre, L. Cuadra, L. Alvarez, L. Alvarez, M. RosaZurera, and F. Lopez-Ferreras, "Automatic sound classification for improving speech intelligibility in hearing aids using a layered structure," in Proceedings of the 7th International Conference Intelligent Data Engineering and Automated Learning (IDEAL '06), vol. 4224 of Lecture Notes in Computer Science, Springer, Burgos, Spain, September 2006.

[9] E. Alexandre, L. Cuadra, L. Álvarez, M. Rosa-Zurera, and F. Lopez-Ferreras, "Two-layer automatic sound classification system for conversation enhancement in hearing aids," Integrated Computer-Aided Engineering, vol. 15, no. 1, pp. 85-94, 2008.

[10] R. O. Duda, P. E. Hart, and D. G. Stork, Pattern Classification, Wiley InterScience, New York, NY, USA, 2001.

[11] E. Alexandre-Cortizo, M. Rosa-Zurera, L. Cuadra, and R. GilPita, "Application of fisher linear discriminant analysis to speech/music classification," in Proceedings of the 120th Audio Engineering Society Convention (AES '06), vol. 2, pp. 16661669, Paris, France, 2006.

[12] P. Nordqvist, Sound classification in hearing instruments, Ph.D. thesis, KTH-S3, Stockholm, Sweden, 2004.

[13] J.-J. Aucouturier and F. Pachet, "Representing musical genre: a state of the art," Journal of New Music Research, vol. 32, no. 1, pp. 83-93, 2003.

[14] E. Guaus and B. Eloi, "A non-linear rhythm-based style classification for broadcast speech-music discrimination," in Proceedings of the 116th Audio Engineering Society Convention (AES '04), Berlin, Germany, May 2004.

[15] H. Demuth and H. M. Beale, Neural Network Toolbox for Use with Matlab, Mathworks, Natick, Mass, USA, 1998.

[16] E. Scheirer and M. Slaney, "Construction and evaluation of a robust multifeature speech/music discriminator," in Proceedings of the IEEE International Conference on Acoustics, Speech and Signal Processing (ICASSP '97), vol. 2, pp. 13311334, 1997.

[17] E. Scheirer, http://labrosa.ee.columbia.edu/sounds/musp/ scheislan.html.

[18] B. Thoshkahna, V. Sudha, and K. R. Ramakrishnan, "A speechmusic discriminator using HILN model based features," in Proceedings of the IEEE International Conference on Acoustics, Speech and Signal Processing (ICASSP '06), vol. 5, pp. 425-428, Toulouse, France, May 2006.

[19] A. L. Berenzweig and D. P. W. Ellis, "Locating singing voice segments within music signals," in Proceedings of the IEEE International Conference on Acoustics, Speech and Signal Processing (ICASSP '01), pp. 119-122, Phoenix, Ariz, USA, 2001.

[20] G. Williams and D. P. W. Ellis, "Speech/music discrimination based on posterior probability features," in Proceedings of the 6th European Conference on Speech Communication and Technology (EUROSPEECH '99), Budapest, Hungary, September 1999.

[21] R. Vicen-Bueno, R. Gil-Pita, M. Utrilla-Manso, and L. ÁlvarezPérez, "A hearing aid simulator to test adaptive signal processing algorithms," in Proceedings of the IEEE International Symposium on Intelligent Signal Processing (WISP '07), pp. 619-624, Piscataway, NJ, USA, October 2007. 